\title{
The Application of Acoustic Myography in Canine Muscle Function and Performance Testing
}

\author{
Christina Fenger ${ }^{1}$ and Adrian P. Harrison ${ }^{1 *}$
}

IVH, Faculty of Health \& Medical Sciences, Copenhagen University, Grønnegaardsvej 7, DK-1870, Frederiksberg C, Denmark

Received: 10 February, 2017; Accepted: 7 March, 2017; Published: 17 March, 2017

*Corresponding author: Adrian P. Harrison, D.Phil.(cantab.), IVH, Faculty of Health \& Medical Sciences, Copenhagen University, Grønnegaardsvej 7 , DK-1870, Frederiksberg C, Denmark, Tel: +45 61462732; E-mail: adh@sund.ku.dk

\begin{abstract}
It is generally known that muscles have the ability to store and convert energy making certain types of movement highly efficient with regard to muscle work. The principle behind this concept is summarized in what has become known as the "spring-mass" model. Whilst evidence of a change in gait from walk to trot has been documented for more than 35 years, quantitative measurements of comparable changes in muscle function remain elusive. In a study involving 11 Labrador dogs, looking at muscle function as assessed by acoustic myography, changes in muscle efficiency/coordination as well as both spatial-(fiber recruitment) and temporal-(firing rate) summation were made for both walk and trot for m.longissimus lumborum and m.gluteus superficialis. In m.gluteus superficialis a $35 \%$ decrease $(\mathrm{P}<0.05)$ in efficiency was noted when dogs changed gait from walk to trot, and both muscles showed a slight yet significant increase in fiber recruitment. However, both muscles showed a significant decrease in firing rate $36 \%$ and $76 \%$ for m.gluteus superficialis and m.longissimus lumborum; $\mathrm{P}<0.05 \& \mathrm{P}<0.01$, respectively) when changing pace from walk to trot. It is concluded that acoustic myography not only enables quantifiable assessment of gait changes with regard to the "spring-mass" model, but that the observed changes in the two muscles measured confirm, as well as advance our understanding of the mechanisms by which reductions in muscle work during steady level movements are achieved.
\end{abstract}

Keywords: Mechanomyography; m.longissimus; m.gluteal; Dog; Muscle contraction; Walk; Trot; Gait change; Spring-mass model

\section{Introduction}

It is known that certain types of muscle contraction associated with specific gaits have the ability to store and convert energy [1,2]. The spring-mass model, as proposed by Blickhan almost thirty years ago, has led to a deeper understanding of the energetic aspects of running or, in the case of dogs, trotting compared to walking [3].

When walking, the center of body mass of a dog is actively raised during the first half of the stance phase to a point above the limbs, and as it subsequently moves back downwards, potential energy is transformed into kinetic energy [4]. In a more recent study, Usherwood and colleagues, showed that dogs, during a slow walk, have relatively stiff limbs, but that as they begin to walk at a faster pace, the limbs begin to impact with the ground and in so doing behave elastically [5]. Clearly, there is then an inverse relation between limb stiffness and an ability to convert potential energy into kinetic energy thus stiffness decreases as speed increases.

In the trot, the body's center of mass behaves differently. Rather than being raised in the first half of the stance phase, it is lowered and the body moves as a wave, often with minimal oscillation in those breeds of dogs that are used to trotting for long periods of time [6]. Here potential energy is converted into elastic energy and stored upon impact with the ground by passive stretching of tendons and muscles, to be released later on in the stance phase. This is the spring-mass model described by Blickhan [3]. The study undertaken by Gregersen and colleagues not only confirms this spring-mass model, it also reveals that the work performed by every joint of the fore- and hind-limbs is more efficient due to a reduced "cost" of physical activity resulting from the release of elastic energy [7]. Indeed, in the trot three quarters of the work performed by dogs is recoverable [7].

Muscles used in locomotion are stretched prior to shortening while producing a force. Compared to concentric contractions (shortening), eccentric contractions (lengthening) result in the highest force with the lowest energy costs due to the higher energy absorption [7,2]. Subsequently, the stored energy can be converted back to kinetic energy in the rebound $[1,2]$. One of the situations in which this is used to great effect is in the very rapid protraction of a horse's fore-limb during periods of galloping, when elastic energy stored in the biceps muscle is released as a burst of energy that results in a catapult action propelling the fore-limb forward [8].

Acoustic Myography (AMG), which directly measures muscle contractions, was recently documented as being a useful diagnostic tool for rapidly determining muscular injury in the veterinary clinic $[9,10]$. Indeed, this technique has been used in the assessment of veterinary clinical diagnosis and monitoring of such dysfunctions or diseases as lameness, kissing spine, crookedness and muscular atrophy as well as post surgery/ trauma monitoring (distance measurement) or retraining/ healthy daily training (http://www.myodynamikequine.com/ 
veterinarian/).

With the recent development of acoustic myography, as a non-invasive and real-time means of monitoring not only the coordination/efficiency of muscle contraction, but also spatial and temporal summation, comes the real possibility of being able to measure and monitor changes in muscle function and performance during a change in gait from walk to trot [11].

This study was designed, to test the following hypotheses; 1) AMG can be used as an informative technique to assess muscle function in dogs during periods of walk and trot, and 2) the AMG signal provides detailed information about muscle efficiency and physiological function when comparing these two gaits.

\section{Methods}

\section{Animals}

A total of 11 Labrador Retrievers were recruited for this study. The owners, which were private individuals, were informed in writing about the AMG technique and the full test programme, and gave their consent for their dog to participate. The dog group comprised 7 females, and the age ranged from 3 to 8 years (mean 6.3 years). The group had an average body mass of $29.7 \mathrm{~kg}$ (range 23 to $37 \mathrm{~kg}$ ), and four of the dogs had been neutered ( 3 females and 1 male). No animals were harmed or stressed as a result of this study.

\section{Acoustic Myography Recordings}

The AMG technique is a non-invasive and pain-free means of recording muscle contractions transdermally $[9,11]$. A dog harness (Julius-K9 IDC, size 1 or 2) was adjusted to fit the dogs in the most appropriate way, so as to fix the CURO unit to the dog (MyoDynamik ApS, Frederiksberg C, Denmark); yet allow a full range of motion.

The muscles longissimus lumborum and gluteus superficialis were measured. The muscle regions were shaved and then cleaned with alcohol (70\%) to disinfect them as well as remove any dirt or hair particles. AMG sensors were placed on the shaved regions above the L4 and L6 for m.longissimus lumborum, and the upper half of the distance between the top of the trochanter major and the lateral apex of the sacrum (in a cranial direction, circa $4 \mathrm{~cm}$, paravertebral) for m.gluteus superficialis. Sensors were covered on the sensing side with acoustic gel (BlueScan - Lina Medical ApS, Glostrup, Denmark) to ensure a good connection with the skin, and attached with flexible bandage (Snogg AS, 4671 Kristiansand, Norway) to the surrounding fur. The sensors were matched in pairs - one pair for m.gluteus superficialis (right and left side) and the same for m.longissimus lumborum. It should be noted that measurements for m.gluteus superficialis were obtained for 10 of the 11 dogs owing to a technical error.

Owing to the nature of the recruitment adopted in this study, dogs were brought for measurement over a wide range of distance and were tested on different dates as well as at different times of the day. Dogs were allowed to rest before the AMG measurements and a short warm-up period involving a few minutes of walking on a lead was performed prior to measurement. The data collection took place outside the laboratory settings at the University Campus on a paved road, except for one dog, which was measured inside the building due to inadequate weather conditions. Two kinds of gaits were measured - walking and trotting. The CURO was switched on and recordings of muscle sounds were carried out transdermally. During measurements, a specially designed app was used with an iPad to follow the muscle signals in real-time, and to ensure that the measurements were without artifacts.

The dog owner was always in contact with the dog to guide it in a straight line during the walking and trotting phases, which happened at a constant pace. The dog was guided with a loose lead minimizing any imbalance in its gait. Each trial took just a few minutes, and the measurements were made in duplicate to minimize inherent errors. The data was stored on the CURO and transferred for data handling at the end of the measurement.

\section{Acoustic Myography Analysis}

When muscle fibers contract, they generate vibrations, and whilst, muscle fiber contractions are not always visible, they do vibrate in active muscles, and as such produce pressure waves, which can be recorded at the level of the skin above a muscle of interest - this is the principle behind acoustic myography, the recording of pressure waves from active muscles transdermally. The CURO system used in this study has been designed to perform a signal analysis, which has purposefully been kept as simple and as pure as possible. Only three parameters are analysed, the degree of efficiency/coordination with which the muscle is used (E-score), the number of active fibers recruited (spatial summation; S-score), and the frequency with which those active fibers are contracted (temporal summation; T-score), all three of which are utilised by the central nervous system when developing force in an active muscle. The AMG data were analyzed in terms of their individual $\mathrm{E}, \mathrm{S}$ and $\mathrm{T}$ scores for each dog, and subsequently, a combined ESTiTM-score was calculated, as the mean of the three individual scores [9].

The E-score represents the period of time a muscle is active relative to periods of inactivity for a set recording interval. The S-score is determined as the signal amplitude in relation to a full $6 \mathrm{~dB}$ signal. Finally, the T-score is determined as the frequency in relation to a Max T value.

Such an AMG signal, occurring in real-time as it does, can be used to assess the level of muscle fatigue, where a stable amplitude and frequency are indicative of a non-fatigued muscle. Muscle performance can also be assessed using the AMG signal. Immediate changes in the S-score, which is a measure of spatial summation, can inform the user as to whether more muscle fibers are being recruited with continuing physical performance [12]. Likewise, a change in the T-score, which represents temporal summation, can provide a minute-to-minute assessment of the frequency with which active fibers are being fired [12]. Finally, the E-score, which represents muscle efficiency and coordination, can be used to assess early fatigue signs [12]. Typically a fall in the E-score, representing relatively more fiber contraction time than relaxed time for a given physical activity, is an early sign of muscle 
fatigue.

\section{Statistical analysis}

Differences between means were tested for statistical significance using GraphPad InStat 3 for Mac (Version 3.0b, 2003; Graph-Pad Inc., La Jolla, CA). Data were initially tested for normal distribution and equal variance, and then subsequently analyzed using a Paired t-test or a Wilcoxon test, as appropriate. Differences between means with a $\mathrm{P}$ value $>0.05$ were considered non-significant. Values are presented as the mean \pm the standard deviation of the mean.

\section{Results}

The dogs responded well to the harness and to the CURO and sensors. They quickly adapted to the attention, and with the aid of their owners help were not only eager to walk and trot, but did so without undue stress or abnormal gait. However, since the dogs recruited in this study were from individual owners, the level of daily activity to which the dogs were exposed could not be standardized. Despite this though, care was taken not to recruit highly trained competition dogs, but rather to include household pets that participated in a regular routine of moderate exercise. A quick analysis of the stride duration for dogs in this study using the AMG data, revealed a stride duration of 0.68 seconds (walking), which is consistent with the Golden Retriever value of 0.67 seconds published by Fischer \& Lilje

\section{m.gluteus superficialis}

The mean E-score for walk was $4.5 \pm 2.4$, whilst for trot it was significantly lower $(2.9 \pm 2.9 ; 35 \%$ decrease $\mathrm{P}<0.05)$. The mean S-score for walk was $6.4 \pm 1.5$, whilst for trot it was significantly lower $(5.5 \pm 1.7 ; 14 \%$ decrease $\mathrm{P}<0.05)$. Finally, the mean T-score for walk was $4.2 \pm 2.3$, whilst for trot it was significantly higher $(5.7 \pm 2.1 ; 36 \%$ increase $\mathrm{P}<0.05)$ (Figure 1 ).


Figure 1: A plot of the mean E-score, S-score and T-score data $(\mathrm{n}=10)$ for walk (Left Panel) and trot (Right Panel) as measured in m.gluteus superficialis of Labrador dogs. Values are presented as the Mean \pm SD, alongside the minimum and maximum values for each data set. Data are for 10 dogs in total.
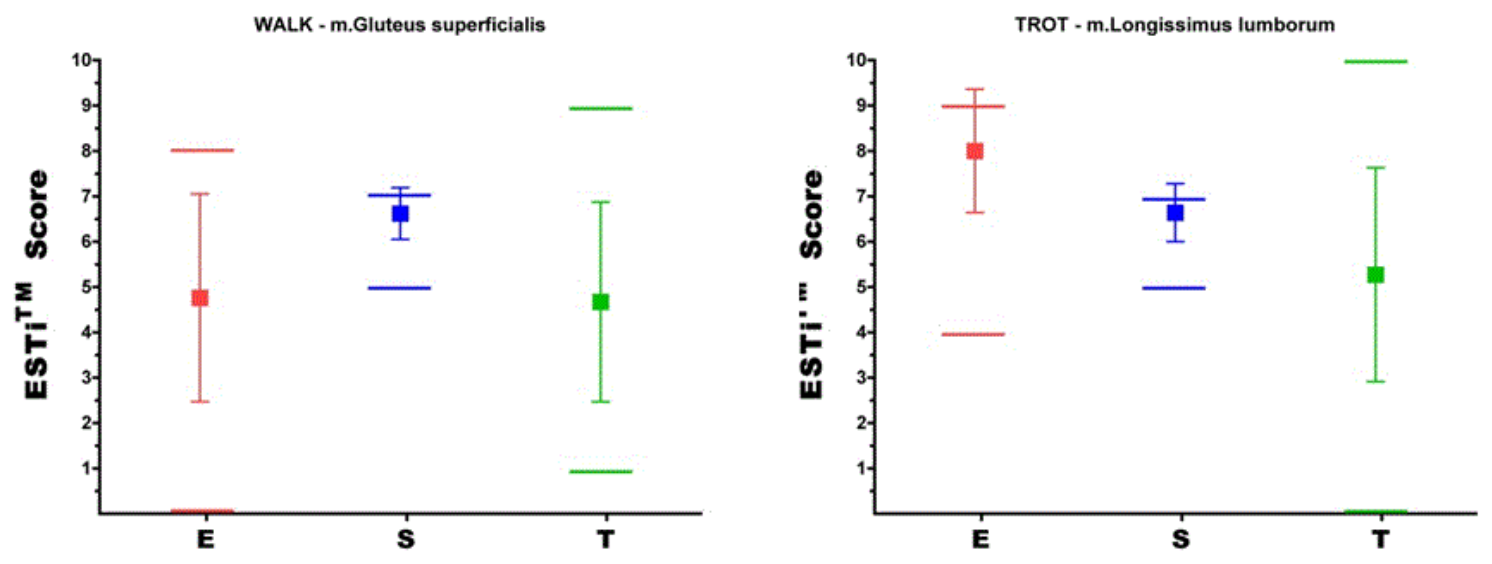

Figure 2: A plot of the mean E-score, S-score and T-score data $(\mathrm{n}=11)$ for walk (Left Panel) and trot (Right Panel) as measured in m.longissimus lumborum of Labrador dogs. Values are presented as the Mean $\pm \mathrm{SD}$, alongside the minimum and maximum values for each data set. Data are for 11 dogs in total. 


\section{m.longissimus lumborum}

The mean E-score for walk was $8.2 \pm 1.1$, whilst for trot it was not significantly changed $(7.7 \pm 1.4 ; \mathrm{P}>0.05)$. The mean S-score for walk was $6.9 \pm 0.4$, whilst for trot it was significantly lower $(6.4 \pm 0.7 ; 7 \%$ decrease $\mathrm{P}<0.01)$. Finally, the mean T-score for walk was $3.0 \pm 2.9$, whilst for trot it was significantly higher $(5.3 \pm 2.4 ; 76 \%$ increase $\mathrm{P}<0.01$ ) (Figure 2).

\section{E, $S$ and T-score changes with altered gait}

With a change of gait from walk to trot, only m.gluteus superficialis showed a decrease in the mean E-score, indicative of less efficient/coordinated contractions. However, both muscles showed a slight decrease in the mean S-score, indicative of a greater degree of fiber recruitment-commensurate with the increased effort of trotting compared with walking.

The mean T-score increased significantly for both muscles when dogs changed their gait from walk to trot, quite dramatically in fact for m.longissimus lumborum. The mean T-score for walk was 3.0 for m.longissimus lumborum, which equates to a firing frequency of $70 \mathrm{~Hz}$, and this decreased to a mean of $47 \mathrm{~Hz}$ for the trot - a 33\% decrease in firing frequency. For m.gluteus superficialis, a similar change was observed since a mean firing frequency of $58 \mathrm{~Hz}$ was recorded for the walk, and a mean firing frequency of $43 \mathrm{~Hz}$ was recorded for the trot - a $26 \%$ decrease in firing frequency.

\section{Discussion}

This study, which has focused on changes in muscle function with a change in gait from walk to trot in dogs, was designed to test two hypotheses. The first hypothesis has been shown to hold true, in that AMG as a technique can be used as an informative means of assessing muscle function in dogs during periods of walk and trot. The second hypothesis was also found to be true, the AMG signal, and in particular the E-score and Tscore reveal details about muscle efficiency and physiological function when comparing walk with trot.

This study, which is the first to use the non-invasive method of acoustic myography to measure changes in muscle function with a change in gait from walk to trot in two canine muscles, has not only revealed that a change in gait results in a decrease in muscle efficiency/coordination with the change in pace, but that there is a slight increase in fiber recruitment to match the increase in force needed to change pace from walk to trot. Perhaps the most interesting finding, however, is that trotting is associated with a lower fiber firing frequency (26-33\%) than walking-resulting in a decrease to a comparable firing frequency of 43-47 Hz for the two muscles measured.

With regard to transfer of force within a muscle to its surroundings, fascia tissue, that is to say bands or sheets of connective tissue (primarily collagen), plays an essential role [14]. Fascia within muscles establishes a network (myofascial complex/network), where individual muscle fibers are surrounded by endomysium, that is to say a thin layer of areolar connective tissue that ensheaths each individual fiber. Bundles of muscle fibers are also surrounded by myofascial connective tissue, referred to as perimysium. Finally, at the periphery of each muscle is another myofascial connective tissue layer, the epimysium, which forms diverse links with surrounding fascia tissue [14]. The forces generated in a muscle fiber are transferred both via the long sarcomere chains that end in a tendinous attachment, but also to a great extent via the sarcomere-myofascial network comprising the endo- peri and epimysium [14]. Indeed, the later myofascial complex serves to transfer the shear forces generated between individual muscle fibers, or the body of a muscle, to the periphery (epimysium). Moreover, this myofascial complex or network provides the parallel elastic element of muscles, which can be used to store elastic energy [15]. When the gait of dogs changes from walk to trot, then the potential and kinetic energy is stored in the myofascial complex as elastic energy. When this elastic energy is released, it results in a passive and energy efficient contraction of the muscle. This can in part explain the lower E-score in m.gluteus superficialis, which serves to indicate the time during which the muscle is inactive during periods of physical activity e.g. less relaxed and more contracted per unit time. The lower E-score found in this study with a change of gait from walk to trot therefore illustrates an increase in the active phase of muscle contraction with this gait, commensurate with a transition towards elastic energy storage within the muscle. Indeed, it has been shown that for $\mathrm{m}$. gluteus superficialis the duration of the stance phase is much longer than the swing phase during walking, and that when dogs change gait to trotting, the duration of the stance phase is markedly reduced and the swing phase is greatly increased [13]. Furthermore, the significant reduction in the frequency of contraction (increase in T-score) in both m. gluteus superficialis and m.longissimus lumborum with a transition from walk to trot also conforms to the idea of stored elastic energy [15].

It has long been known that quadrupedal species convert potential energy and kinetic energy into elastic energy in spring elements of the limbs and trunk to regain some expended energy later during limb support $[16,17]$. In this way, limb and trunk spring compliance enables the storage and return of elastic energy to reduce the work of muscle contraction. Indeed, it is now generally accepted that the mass-spring elastic energy savings achieved during trotting by most quadrupeds are an evolutionary adaptation designed to minimize muscle work during steady level movement [17]. The T-score data from this study supports the mass-spring elastic energy model, in that a reduction in firing frequency with a change of pace from walk to trotting would serve to reduce and minimize muscle work during the steady level movement of trotting. Furthermore, for the first time, these data serve to quantify these changes in terms of muscle contraction.

One of the first studies to examine the muscle contraction activity of dog limb muscles as a function of speed and gait was Goslow and colleagues [18]. They used sEMG to assess the timing of electrical activity in active muscles and related it to the phases of individual gaits as well as limb joint angle. Whilst 
their meticulous work shows the precise timing of muscle contractions, they make no assessment of the firing frequency of the sEMG signal, and thereby provide no comparative data on muscle firing rate. However, these authors discovered functional aspects relating to m.gluteus superficialis. They reported that during walk the muscle served to extend the hip, by some $30 \mathrm{o}$, and that it became active just before the foot touched the ground and remained active for $48 \%$ of the stride period [18]. Interestingly, during trot, m.gluteus superficialis became active before the foot touched the ground but was active for only $27 \%$ of the stride period, and extension of the hip was only 150 . Sadly no such data are available for m.longissimus lumborum, which was not measured by Goslow and colleagues [18].

In order to conserve energy during trotting it is clearly necessary to reduce and minimize muscle work. Indeed one way of doing just that is to reduce the period of active contraction, as observed by Goslow and colleagues a little over 35 years ago [18]. A reduction in active muscle contraction from $48 \%$ of the stride period to just $27 \%$ when going from the walk to the trot must surely serve to reduce muscle work. However, returning to the spring-mass model and viewed in the light of the recent findings of Usherwood and colleagues, limb stiffness decreases as speed increases $[3,5]$. In other words, recent findings that dogs during walk have relatively stiff limbs, but during trot their limbs behave elastically, must also hint that other changes are at work. For the spring-mass model to work efficiently during walk, the center of body mass needs to be lifted above the limbs and then allowed to drop back to its starting level - yet this also requires that the body mass remains relatively rigid in order for this to occur. The present findings of a low T-score (3.0) for m.longissimus lumborum, equating to a firing frequency of $70 \mathrm{~Hz}$, is in keeping with this. Likewise, with an increase in speed from walk to trot there is a need for the limb muscles, and we would also suggest for the back muscles, to act more elastically. The later could only be achieved if the muscles were allowed to relax a little, something that would necessitate a lower firing rate - which is precisely what the present findings reveal for both m.longissimus lumborum and m.gluteus superficialis. It is likewise interesting that both these muscles have a similar firing rate during the trot (43-47 Hz). Thus, when combined with the reduced period of active muscle contraction as reported by Goslow and colleagues this change in the $\mathrm{T}$-score equating to a lower muscle firing rate with a change from walk to trot, must also serve to reduce and minimize muscle work during such steady level movements as trotting, as well as facilitate the storage and release of elastic energy [18].

\section{Conclusion}

Overall, the AMG technique is a promising non-invasive method to assess muscle function changes relating to elastic strain energy adaptations in muscles during a change of pace from walk to trot in dogs.

\section{Acknowledgement}

The authors wish to thank the individual dog owners for their time and interest in this study, and both Kiara Heide Salomons and Anja Harder from MyoDynamik ApS for their technical support. In addition, we wish to thank Drs. Else Marie Bartels and Vibeke Sødring Elbrønd for reading this manuscript and proposing improvements to the text.

\section{Declarations}

Conflict of Interest: A.H. is currently trying to commercialize the AMG recording system and is establishing a company to cover the costs of future development. This does not represent a conflict of interest as the protocol for this study was designed solely by C.F, and the recordings were undertaken independently by C.F.

\section{Ethical approval}

NA - no animals were harmed or stressed as a result of this study which involved solely non-invasive techniques.

\section{References}

1. Alexander RM, Bennet-Clark HC. Storage of elastic strain energy in muscle and other tissues. Nature. 1977; 265(5590):114-117.

2. Lindstedt SL, Reich TE, Keim P, LaStayo PC. Do muscles function as adaptable locomotor springs? J Exp Biol. 2002;205(Pt 15):2211-2216.

3. Blickhan R. The spring-mass model for running and hopping. J. Biomech. 1989; 22(11-12):1217-1227.

4. Alexander RM. Mechanics of bipedal locomotion. In: Davies, PS (ed): Perspectives in Experimental Biology. Oxford, Pergamon Press. 1976;493-504.

5. Usherwood JR, Williams SB, Wilson AM. Mechanism of dog walking compared with passive, stiff-limbed, 4-bar linkage model, and their collisional implications. J. Exp. Biol. 2007;210(Pt 3):533-540.

6. Jayes AS, Alexander RM. Mechanics of locomotion of dogs (Canis familiaris) and sheep (Ovis aries). J. Zool. 1978; 185 Pt 3:289-308.

7. Gregersen CS, Silverton NA, Carrier DR. External work and potential for elastic storage at the limb joints of running dogs. J Exp Biol. 1998;201(Pt 23):3197-3210.

8. Wilson AM, Watson J, Lichtwark GJ. Biomechanics: A catapult action for rapid limb protraction - Energy bursts from a horse's elastic biceps muscles provide power for a flat-out gallop. Nature. 2003;421(6918):35-36. doi: $10.1038 / 421035 \mathrm{a}$.

9. Harrison AP. A more precise, repeatable and diagnostic alternative to surface electromyography - an appraisal of the clinical utility of acoustic myography. Clin Physiol Funct Imaging. 2017. doi: 10.1111/cpf.12417.

10. Riis KH, Harrison AP, Riis-Olesen K. Non-invasive assessment of equine muscular function: A case study. Open Vet J. 2013;3(2):80-84.

11. Harrison AP, Danneskiold-Samsøe B, Bartels EM. Portable acoustic myography - a realistic noninvasive method for assessment of muscle activity and coordination in human subjects in most home and sports settings. Physiol. Rep. 2013;1(2). doi:10.1002/phy2.29. 
12. Hunt CC, Kuffler SW. Motor innervation of skeletal muscle: Multiple innervation of individual muscle fibres and motor unit function. J. Physiol. 1954;126(2):293-303.

13. Fischer MS, Lilje KE. Dogs in motion. Second edition. Germany: Dortmund: VDH Service; 2014.

14. Huijing PA. Muscle as a collagen fiber reinforced composite: a review of force transmission in muscle and whole limb. J Biomech. 1999;32(4):329345 .

15. Schleip R, Müller DG. Training principles for fascial connective tissues: Scientific foundation and suggested practical applications. J Bodyw Mov Ther. 2013;17(1):103-115. doi.org/10.1016/j.jbmt.2012.06.007

16. Cavagna GA, Kaneko M. Mechanical work and efficiency in level walking and running. J. Physiol. 1977;268(2): 467-481.

17. Biewener AA. Patterns of mechanical energy change in tetrapod gait: pendula, springs and work. J Exp Zool A Comp Exp Biol. 2006;305(11):899911.

18. Goslow GE Jr, Seeherman HJ, Taylor CR, McCutchin MN, Heglund NC. Electrical activity and relative length changes of dog limb muscles as a function of speed and gait. J. Exp. Biol. 1981;94:15-42. 